\title{
A Study on the Distributed Antenna Based Heterogeneous Cognitive Wireless Network Synchronous MAC Protocol
}

\author{
Lian-Fen Huang, ${ }^{1}$ Sha-Li Zhou, ${ }^{1}$ Yi-Feng Zhao, ${ }^{1}$ and Han-Chieh Chao ${ }^{2,3}$ \\ ${ }^{1}$ Department of Communication Engineering, Xiamen University, Xiamen, Fujian 361005, China \\ ${ }^{2}$ Institute of Computer Science \& Information Engineering and Department of Electronic Engineering, \\ National Ilan University, I-Lan, Taiwan \\ ${ }^{3}$ Department of Electrical Engineering, National Dong Hwa University, Hualien, Taiwan \\ Correspondence should be addressed to Yi-Feng Zhao; zhaoyf@xmu.edu.cn
}

Received 1 September 2014; Accepted 1 September 2014

Academic Editor: Ilsun You

Copyright (C) 2015 Lian-Fen Huang et al. This is an open access article distributed under the Creative Commons Attribution License, which permits unrestricted use, distribution, and reproduction in any medium, provided the original work is properly cited.

\begin{abstract}
This paper introduces distributed antennas into a cognitive radio network and presents a heterogeneous network. The best contribution of this paper is that it designs a synchronous cognitive MAC protocol (DAHCWNS-MAC protocol: distributed antenna based heterogeneous cognitive wireless network synchronous MAC protocol). The novel protocol aims at combining the advantages of cognitive radio and distributed antennas to fully utilize the licensed spectrum, broaden the communication range, and improve throughput. This paper carries out the mathematical modeling and performance simulation to demonstrate its superiority in improving the network throughput at the cost of increasing antenna hardware costs.
\end{abstract}

\section{Introduction}

DAS (distributed antenna system) is used as an extension of the outdoor cellular mobile system in early stage, which is widely used for indoor or blind spot coverage. However the research of MIMO (multiple input multiple output) technology has become more and more sophisticated, which provides a broader space for the further development of DAS. This network structure can improve the wireless signal covering ability and system capacity and obtain high power efficiency. Because of these advantages, the DAS has been considered to be a key way of multiple antennas accessing in future mobile communication. Most current researches on the distributed antenna are focused on how distributed antennas can be used in the cellular mobile network physical layer to improve network capacity. Introducing DAS into WLAN (wireless local area network) can fully utilize its physical advantages to greatly improve the performance of WLAN, which is an attractive research area in the future. However there is scant literature on how distributed antenna can be used in WLANs. Reference [1] thinks that the fixed channel allocation method is not flexible and fair enough for multicell WLAN. New systems replace APs (access points) with distributed antennas using a control center. The control center dynamically adjusts the channel assignment scheme according to a judgment standard determined by the throughput, fairness, and other factors. Reference [2] designs the control center internal structure used in the distributed antenna WLAN system. The author thinks that each channel needs a corresponding processing unit. The more nodes the antenna services, the more channels it needs. The antenna therefore allocated more processing units.

This paper studies distributed antenna applications in the MAC (media access control) layer and designs a distributed antenna-based synchronous MAC protocol to sense the spectrum and transmit data.

Numerous literatures have studied spectrum sensing, producing some novel algorithms. References $[3,4]$ proposed a multitaper method (MTM) to detect the spectrum, with the advantages of low complexity and high detection accuracy. References $[5,6]$ proposed a detection method based on the signal covariance matrix. The ratio of two statistics is used to judge if the primary user appears. References $[7,8]$ studied the generalized likelihood ratio test method. To eliminate 
the influence of noise uncertainty, it first uses the maximum likelihood algorithm to estimate the unknown noise power and then completes the detection algorithm.

In addition to spectrum sensing we can further use distributed antennas to locate the primary user and adopt different access methods according to positioning information. Reference [9] studied the power allocation problem under overlay/underlay hybrid access mechanism. Reference [10] introduced the distributed antenna into the cognitive radio network. It utilizes the distributed antenna to sense the spectrum and locate the primary user. The author designed an asynchronous cognitive MAAC-MAC protocol (multiantenna asynchronous cognitive MAC).

This paper focuses on how the distributed antenna can be used in data transmission and does not explore its usage in spectrum sensing and positioning. In future studies we can continue this research in different directions.

In order to design an appropriate network architecture for DAS, this paper studies the hybrid wireless networks. Many documents have studied hybrid wireless networks [11-14]. Adding AP/BSs (access point/base stations) into an ad hoc network can combine the network advantages with ad hoc infrastructure. Currently related literatures are focused on a cellular network and ad hoc network combination. In [15] the whole area is partitioned into many cells and all cells use the TDMA (time division multiple access) scheme. The author proposes two hybrid routing strategies that combine direct transmission (ad hoc mode) and forwarding data through base stations (infrastructure mode). Reference [16] proposes a protocol to judge whether data needs to be forwarded or not in the hybrid network.

This paper introduces the distributed antenna into the cognitive radio network and designs a heterogeneous network consisting of an ad hoc network and a sparse network of distributed antennas. The new network utilizes the distributed antennas to sense the spectrum and transmit data. We design a synchronous cognitive MAC protocol (DAHCWNS-MAC protocol) that can improve the sensing performance and also broaden the communication range to increase the throughput compared to the original single-hop network.

\section{Heterogeneous Network Model}

2.1. Communication Scenario. The distributed antenna layout is shown in Figure 1. Distributed antennas are uniformly placed throughout the region. Seven antennas are used to cover the entire communication scenario. The seven antennas are connected to a control center via an optical fiber. Distributed antennas forward data only with the other complex processing work completed by the control center.

2.2. Network Architecture. In an $802.11 \mathrm{DCF}$ network with infrastructure all data is forwarded by the access point (AP). After adding distributed antennas the original AP coverage is divided into seven smaller cells, each of which is covered by an antenna. Within the coverage of each antenna the distance between nodes can be regarded as one hop and

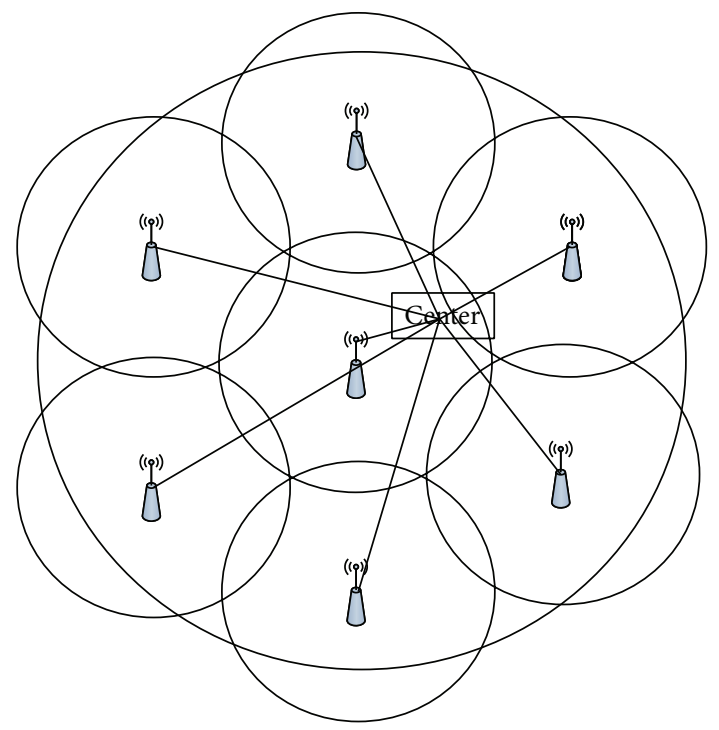

FIgURE 1: The layout of distributed antennas.

they can send data to each other directly. A node cannot communicate directly with its destination node when its destination node is not located in the same cell. In this case distributed antennas are used to forward data. We assume that nodes are uniformly placed in the region and each node accesses its nearest antenna. When a node and its destination node are located in the same antenna coverage they can send data directly. However, if a node and its destination node are not located in the same cell, they have to use distributed antennas to forward data. This is a heterogeneous network consisting of an ad hoc mode and infrastructure mode.

This heterogeneous network combines the advantages of two different networks. The network with infrastructure is easy to manage and does not have to exchange messages among nodes as in the ad hoc network. This reduces the extra overhead. It can also broaden the communication range through forwarding data by infrastructure. Under the ad hoc mode direct data transmission decreases the time consumed by forwarding, so the throughput can be improved.

The DAHCWNS-MAC protocol is designed for cognitive radio networks where nodes use idle licensed bands to communicate. The spectrum sensing is an important part of the protocol. What is different from the previous cognitive MAC protocols is that the DAHCWNS-MAC protocol uses distributed antennas instead of nodes to sense the spectrum. Results at all the antennas will be submitted to the control center for a final judgment result. The idle bands will be allocated to nodes by the control center. Nodes do not have to sense the spectrum by themselves as in the selforganized network, which reduces the exchanging sensing results overhead and decreases the node hardware requirements. Furthermore, the sensing performance is improved while utilizing the distributed antennas' macrodiversity.

We can utilize distributed antennas to locate primary users and adopt overlay/underlay hybrid access schemes through power control for more effective licensed band usage. 


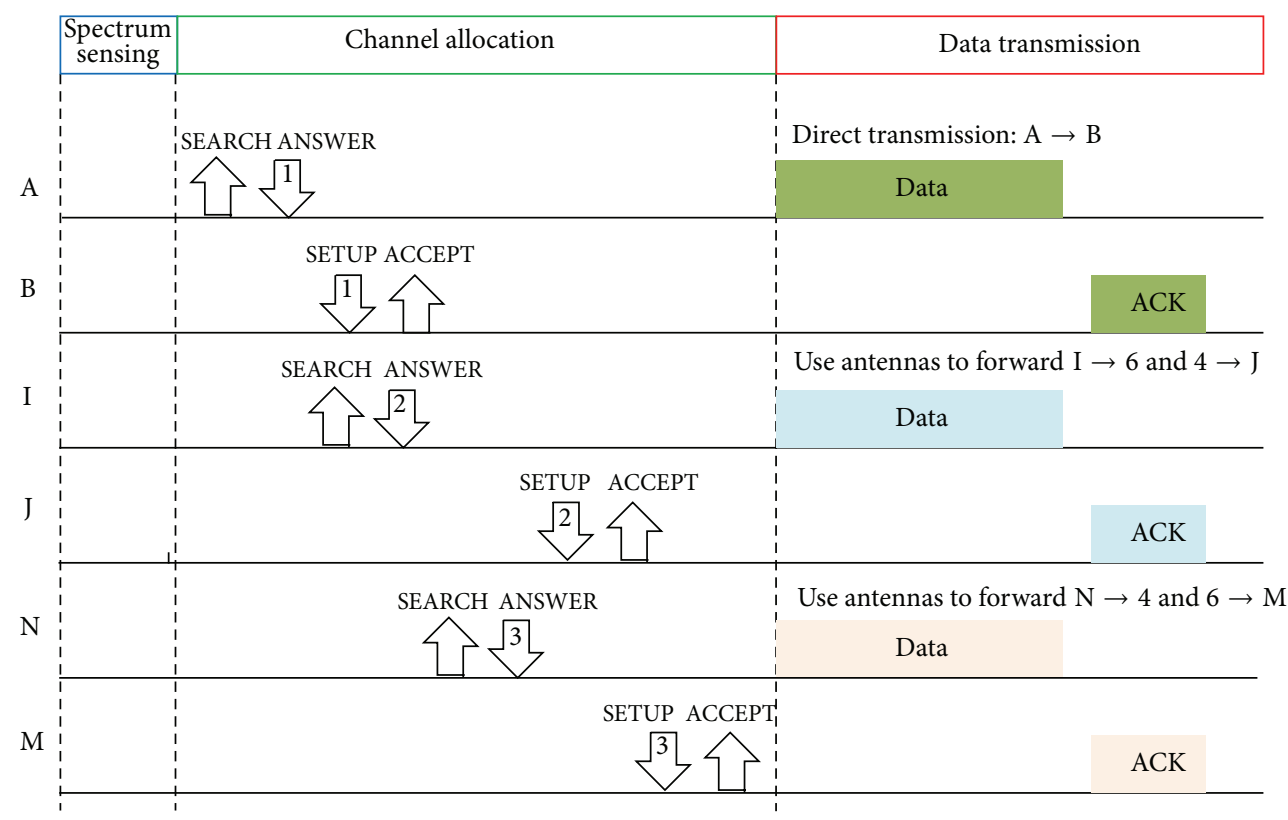

FIGURE 2: The time frame of DAHCWNS-MAC protocol.

Section 1 introduces some novel sensing algorithms and hybrid access schemes that are very useful in completing the DAHCWNS-MAC protocol in future research. Because this paper aims mainly at exploring how distributed antennas are used for data transmission, we no longer repeat the spectrum sensing and positioning parts of the protocol in the next article.

\section{The Distributed Antenna Based Heterogeneous Cognitive Wireless Network Synchronous MAC Protocol (DAHCWNS-MAC Protocol)}

In this section the DAHCWNS-MAC protocol will be described in detail.

3.1. Assumptions. Before presenting the specific protocol some necessary assumptions are summarized as follows.

(a) There are $N+1$ licensed channels for use, all of which have the same bandwidth. Since no overlap occurs among channels, packets transmitted on different channels will not affect each other. The control center knows how many licensed channels can be used in advance.

(b) One of $N+1$ licensed channels is used as the control channel. This can be the unlicensed band in practice and thus free from interference from the primary users.

(c) Each CU (cognitive user) is equipped with a single cognitive radio. This radio can either transmit or receive, but it cannot do both simultaneously. (d) All antennas can forward data correctly. The control center has different processing units corresponding to different antennas, so it can parallel process data from different antennas. The control center also knows which nodes exist within the antenna coverage.

(e) All nodes are strictly synchronous. They always start and finish a beacon interval at the same time.

(f) Distributed antennas can sense the spectrum precisely to obtain all idle channels.

3.2. Protocol Design. The DAHCWNS-MAC protocol is a synchronous MAC protocol. The whole time can be divided into frames with fixed length. The time frame can be separated into three parts: sensing, channel allocation, and transmission phases. The DAHCWNS-MAC time frame is depicted in Figure 2.

(1) The first part of the DAHCWNS-MAC protocol is the sensing phase. Distributed antennas sense spectrum during this period. Each antenna detects $\mathrm{N}$ channels independently and submits its result to the control center. The control center will get final judgment result through data fusion and allocates idle channels to nodes to communicate in the next part of the protocol.

(2) The second part of the DAHCWNS-MAC protocol is the channel allocation phase. This phase is based on the CSMA/CA mechanism. All nodes contend with each other for the right to use channels. Since this network is a heterogeneous network it is necessary to determine whether nodes need distributed antennas to forward data. The specific process is as follows. Nodes that have data to transmit contend to send SEARCH frames to their nearest antenna on the control channel. If the competition succeeds, the corresponding antenna will submit the frame to the control center and then stores it. At the same time the control center 
will reply with an ANSWER frame to the node which includes the number of allocated channels (randomly selected from the remaining channels until no idle channels remain). The above is the uplink part of this phase.

Upon receiving the SEARCH frame the control center will find the number of distributed antennas to which the destination node belongs and check if the sending and receiving nodes are located in the same antenna coverage area. If so, then in the next data transmission phase there is no need for distributed antennas to forward data. The sending and receiving nodes can communicate directly. If not, data packets are forwarded by distributed antennas. The control center records the result if a pair of nodes need data forwarding and in the next data transmission phase it can use the result to judge whether to forward data packets from this node. Next the control center checks if the antenna to which the receiving node belongs is idle. If the antenna is idle a SETUP frame will be sent to the receiving node immediately which contains the same channel number as the ANSWER frame. Otherwise, the SETUP frame will be sent until the antenna becomes idle. Upon the receiving node getting the SETUP frame it will reply with an ACCEPT frame, which means the handshake is completed. The above is the downlink part of the channel allocation phase.

Because the uplink and downlink capacity are unbalanced in networks with infrastructure, to ensure downlink transmission completion, the protocol does not use a competition scheme during the downlink, which means the nodes do not need to perform backoff. Specifically, there is no need for backoff before sending the SETUP frame.

The transceiver node pair completes any message exchange necessary for the data transmission phase through a four-way handshake. As shown in Figure 3 the four-way handshake includes two parts: the uplink part and downlink part.

The DAHCWNS-MAC protocol makes the collision domain narrow to the antenna coverage. Cells covered by different antennas are independent and do not affect each other. Each antenna can work in parallel with others, which means different antennas can be in different transmission/receiving stages at the same time.

All operations during this phase are carried out on the control channel. The control channel may also be used for data transmission.

(3) The third part of the DAHCWNS-MAC protocol is the data transmission phase. The transceiver node pair which completes the four-way handshake can send data on the channel allocated to them. If they need help from distributed antennas in forwarding data the corresponding antenna will forward their data automatically. Otherwise, they will communicate directly until this time frame ends. A transceiver node pair that does not complete the four-way handshake cannot enter the data transmission phase.

The time frame is divided into three parts. As illustrated there are three pairs of nodes communicating: A to B, I to J, and $\mathrm{N}$ to $\mathrm{M}$. For example, $\mathrm{A}$ wants to communicate with $\mathrm{B}$ and it sends SEARCH frame to the center. Then the center replies with ANSWER frame which allocates channel 1 to A. At the same time the center sends SETUP frame to B to tell B that

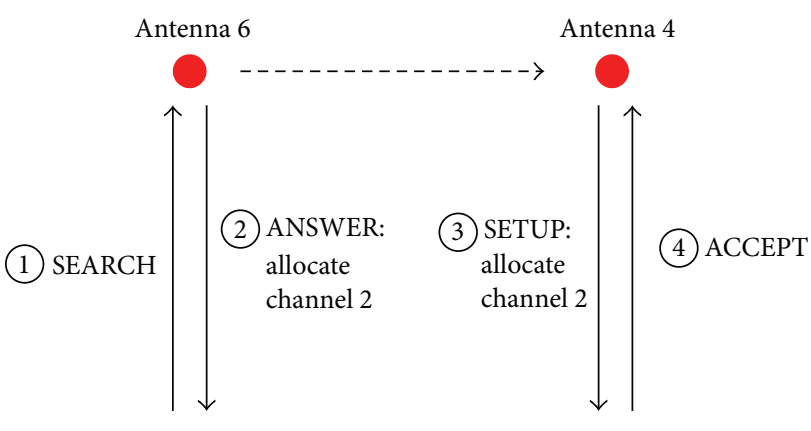

Node I

Node J

FIgURE 3: The four-way handshake.

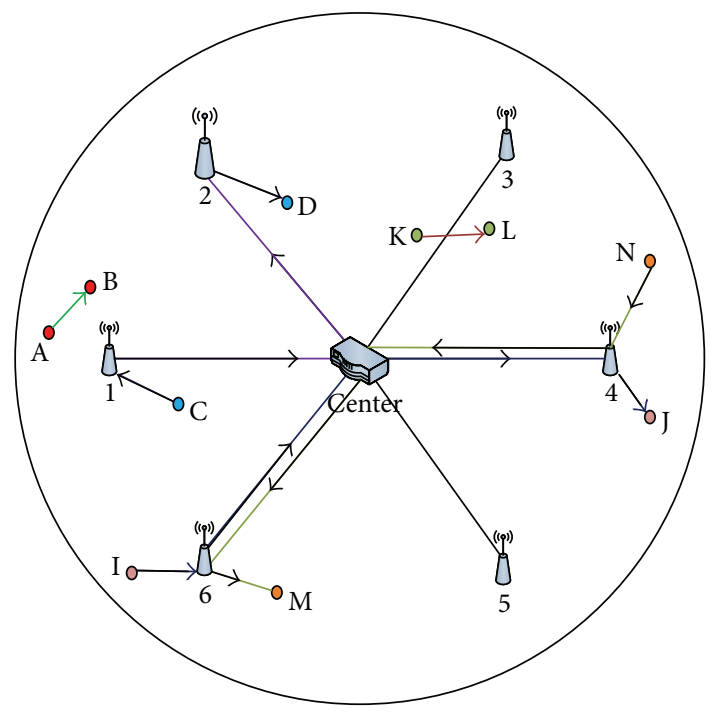

Figure 4: Transmission example.

the allocated channel is 1 and then B replies with ACCEPT frame to complete four-way handshake. Because nodes A and $\mathrm{B}$ are in one-hop distance, they can transmit directly in channel 1. I and J communicate on channel 2 through antenna forwarding. $\mathrm{N}$ and $\mathrm{M}$ communicate on channel 3 through antenna forwarding.

A specific transmission case is presented in Figure 4. For example, nodes A and B are located in antenna 1 coverage area. They can communicate directly because the control center allocated channel 1 to them. Nodes I and J are located in different antenna coverage areas, so they need antennas 4 and 6 to forward data with channel 2 assigned to them.

This figure uses the communication process between nodes I and J to explain how the four-way handshake works. The first step: node I sends SEARCH frame to antenna 6 . The second step: the center replies with ANSWER frame which allocates channel 2 to I. The third step: at the same time antenna 4 sends SETUP frame to node J to tell J that the allocated channel is 2 . The last step: node J replies with ACCEPT frame to complete four-way handshake.

3.3. The DAHCWNS-MAC Protocol Model. In this section a mathematical model for the DAHCWNS-MAC is presented to theoretically calculate its saturated throughput. 
Since only transceiver nodes that complete a handshake can enter the data transmission phase, the key in calculating throughput is to calculate how many pairs of transceiver nodes complete the handshake during the channel allocation phase. The channel allocation phase is divided into uplink and downlink parts. How many times the downlink part is finished represents how many pairs of transceiver nodes complete the handshake. Therefore the objective is to calculate the downlink part throughput during the channel allocation phase.

Whether frames are sent in the downlink part depends on the uplink part. The SETUP frame is sent only when the SEARCH frame has been successfully received. Since seven distributed antennas operate independently, the uplink and downlink transmissions in different cells are independent of each other. Therefore, the average throughput for the seven cells should be the same. It is feasible to calculate the downlink throughput of one cell first and then multiply it by seven.

We assume that sending nodes always have data to transmit. Nodes contend to send frames in uplink and the transmission probability in each slot can be calculated using the two-dimension Markov chain model. Since there is no competition during downlink, the downlink transmission probability is equal to the probability of generating downlink data.

Several necessary variables are defined as follows. $\tau^{\text {up }}$ and $\tau^{\text {down }}$ represent the transmission probability in each slot of uplink and downlink, respectively. The average number of sending nodes in each cell is $n$.

In 802.11DCF network, the transmission probability in each slot can be calculated using the two-dimension Markov chain model according to [17]. The transmission probability of the uplink part should be the same. So

$$
\tau^{\mathrm{up}}=\frac{2(1-2 p)}{(1-2 p)(\mathrm{CW}+1)+p \mathrm{CW}\left[1-(2 p)^{m}\right]},
$$

where $\mathrm{CW}$ is the size of the smallest contention window and $m$ is the maximum backoff stage. The collision probability of uplink is

$$
p=1-\left(1-\tau^{\text {up }}\right)^{n-1}\left(1-\tau^{\text {down }}\right) .
$$

There is no need in downlink for backoff before sending data. Frames are sent out as soon as the channel is idle. Therefore, the transmission probability of downlink is equal to the probability of generating downlink data. The probability depends on the uplink transmission success. Only when the uplink transmission succeeds, which means the SEARCH frame has been successfully stored in center cache, will the corresponding exit antenna have data to send. So the downlink transmission probability is assumed to be equal to the probability of successful uplink transmission:

$$
\tau^{\text {down }}=p_{s}^{\text {up }} p_{\text {tr }}^{\text {up }}=n \tau^{\text {up }}\left(1-\tau^{\text {up }}\right)^{n-1}\left(1-\tau^{\text {down }}\right) .
$$

Combining (1), (2), and (3), the $\tau^{\text {up }}$ and $\tau^{\text {down }}$ can be solved.

According to [18] the probability that at least one uplink frame is in transmission at some slot is

$$
p_{\text {tr }}^{\text {up }}=1-\left(1-\tau^{\text {up }}\right)^{n} .
$$

Under the case that uplink frames exist which are in transmission, the probability that only one uplink frame is being transmitted is

$$
p_{s}^{\text {up }}=\frac{n \tau^{\text {up }}\left(1-\tau^{\text {up }}\right)^{n-1}\left(1-\tau^{\text {down }}\right)}{1-\left(1-\tau^{\text {up }}\right)^{n}} .
$$

Likewise, under the case that downlink frames exist which are in transmission, the probability that only one downlink frame is being transmitted is

$$
p_{s}^{\text {down }}=\frac{\tau^{\text {down }}\left(1-\tau^{\text {up }}\right)^{n}}{\tau^{\text {down }}} .
$$

Four possible states exist in one slot as follows.

(1) The channel is idle and the probability is $\left(1-p_{\mathrm{tr}}^{\mathrm{up}}\right)(1-$ $\left.\tau^{\text {down }}\right)$. The length of a slot is $\sigma$.

(2) The uplink frame is transmitted successfully and the probability is $p_{s}^{\text {up }} \tau^{\text {down }}$. The average transmission time is $T_{s}^{\text {up }}$.

(3) The downlink frame is transmitted successfully and the probability is $p_{s}^{\text {down }} \tau^{\text {down }}$. The average transmission time is $T_{s}^{\text {down }}$.

(4) When a collision happens the probability is $p_{\mathrm{tr}}^{\mathrm{up}}(1-$ $\left.p_{s}^{\text {up }}\right)+\tau^{\text {down }}\left(1-p_{s}^{\text {down }}\right)-p_{\text {tr }}^{\text {up }} \tau^{\text {down }}$. The average time is $T_{c}$.

The downlink throughput is

$$
s=\frac{\tau_{\text {down }} P_{s}^{\text {down }} E^{\text {down }}}{\left(1-P_{\text {tr }}^{\text {up }}\right)\left(1-\tau_{\text {down }}\right) \sigma+P_{\text {tr }}^{\text {up }} P_{s}^{\text {up }} T_{s}^{\text {up }}+\tau_{\text {down }} P_{s}^{\text {down }} T_{s}^{\text {down }}+\left(P_{\text {tr }}^{\text {up }}\left(1-P_{s}^{\text {up }}\right)+\tau_{\text {down }}\left(1-P_{s}^{\text {down }}\right)-P_{\text {tr }}^{\text {up }} \tau_{\text {down }}\right) T_{c}} .
$$

The denominator of the above formula is the average length of one slot where the downlink transmission occupies a proportion:

$$
r=\left(\tau_{\text {down }} P_{s}^{\text {down }} T_{s}^{\text {down }}\right)
$$

$$
\begin{aligned}
& \times\left(\left(1-P_{\text {tr }}^{\text {up }}\right)\left(1-\tau_{\text {down }}\right) \sigma+P_{\text {tr }}^{\text {up }} P_{s}^{\text {up }} T_{s}^{\text {up }}\right. \\
& \quad+\tau_{\text {down }} P_{s}^{\text {down }} T_{s}^{\text {down }}
\end{aligned}
$$




$$
\begin{aligned}
+ & \left(P_{\mathrm{tr}}^{\mathrm{up}}\left(1-P_{s}^{\mathrm{up}}\right)+\tau_{\text {down }}\left(1-P_{s}^{\text {down }}\right)\right. \\
& \left.\left.-P_{\mathrm{tr}}^{\mathrm{up}} \tau_{\text {down }}\right) T_{c}\right)^{-1} .
\end{aligned}
$$

So the total number of transceiver nodes that can complete a handshake during the channel allocation phase is

$$
\text { num }=7 * \text { CA_window length } \times \frac{r}{T_{s}^{\text {down }}}
$$

where CA_window_length is the length of the channel allocation phase.

The final saturation throughput is

$$
\begin{aligned}
s= & \frac{1}{7} \times \text { num } \times \frac{\text { data_window_length }}{T_{\text {data }}} \times l \times 8+\frac{6}{7} \\
& \left.\times \text { num } \times \frac{\text { data_window_length }}{2 \times T_{\text {data }}} \times l \times 8\right) \\
& \times(\text { interval length })^{-1},
\end{aligned}
$$

where data_window_length is the length of the data transmission phase, $T_{\text {data }}$ is the transmission time of a data packet, $l$ is the real payload in a data packet, and interval_length is the length of a time frame.

As nodes are randomly placed, the probability that sending and receiving nodes are located in the same cell is $1 / 7$ at which time they can send data directly. However, the probability that sending and receiving nodes are located in a different cell is $6 / 7$ at which time distributed antennas should help in forwarding data. The time required for forwarding data is doubled compared to that of sending directly.

\section{Performance Simulation and Analysis}

In this section we present the DAHCWNS-MAC protocol simulation results. We assume a time frame is fixed at $100 \mathrm{~ms}$ and the state of licensed channels changing at the beginning of each time frame and remaining unchanged until the time frame ends up. The related parameters are shown in Table 1 . This paper uses $C$ language and MATLAB 7.0 as simulation tools. The simulation results come from $\mathrm{C}$ language programming and the theoretic results come from MATLAB programming.

4.1. The Comparison of Mathematical Model and Simulation Throughput. The channel allocation phase length is fixed to $10 \mathrm{~ms}$ and the number of idle channels is unlimited (all the transceiver nodes pairs which complete four-way handshake can be assigned a channel). A comparison between the mathematical model and simulation results is shown in Figure 5. We can see that the outcome for the two is quite close. This demonstrates the accuracy of the mathematical model. As the number of sending nodes increases, the throughput first increases and then decreases. This is because when the number of nodes increases the collision probability increases as well. The increasing collision probability results in a
TABLE 1: The main simulation parameters.

\begin{tabular}{lc}
\hline Data channel rate & $1 \mathrm{Mb} / \mathrm{s}$ \\
Control channel rate & $1 \mathrm{Mb} / \mathrm{s}$ \\
Transmission delay & $1 \mathrm{us}$ \\
DIFS & $50 \mathrm{us}$ \\
SIFS & $10 \mathrm{us}$ \\
Slot time & $50 \mathrm{us}$ \\
Maximum backoff state $(m)$ & 4 \\
Data packet length & $512 \mathrm{bytes}$ \\
Time frame length & $100 \mathrm{~ms}$ \\
Simulation time & $100 \mathrm{~s}$ \\
\hline
\end{tabular}

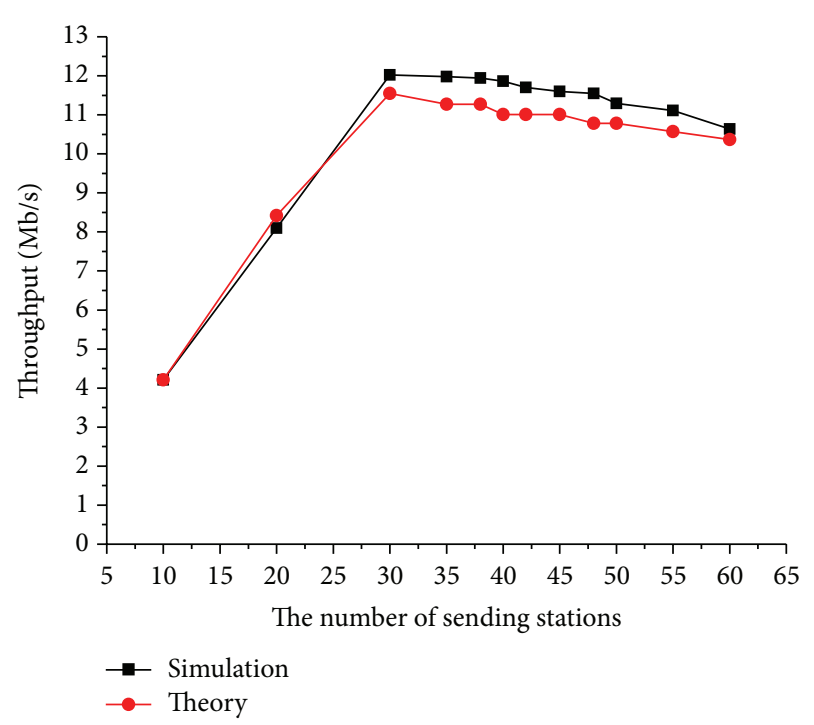

FIGURE 5: The saturation throughput of mathematical model and simulation.

decreasing number of nodes that can complete a handshake and enter the transmission phase. Therefore, the throughput eventually decreases.

4.2. The Relationship between Throughput and Channel Allocation Phase Length. Now let us analyze the channel allocation phase length effect on the saturation throughput. With 50 sending stations and 50 licensed channels, as shown in Figure 6, the saturation throughput increases as the time increases from $5 \mathrm{~ms}$ to $20 \mathrm{~ms}$ when the primary users' activity rate is 0 . However, the saturation throughput decreases after $20 \mathrm{~ms}$. This is because the number of nodes that complete a handshake is limited when the length is smaller than $20 \mathrm{~ms}$. As the length becomes larger, the number of nodes that can complete a handshake to get a channel becomes larger, which results in an increase in the saturation throughput. However, the length of the time frame is fixed in the DAHCWNSMAC protocol, which means the data transmission time will decrease when the channel allocation time increases. As the throughput reaches peak when the time is $20 \mathrm{~ms}$ because all transceiver nodes can get idle channels at this moment, increasing the channel allocation time results in the data 


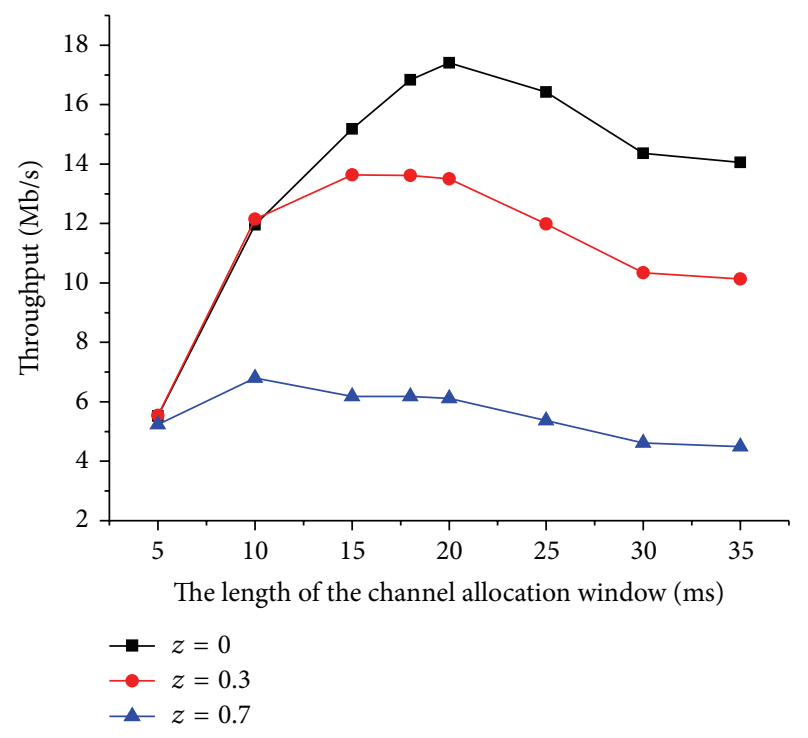

Figure 6: The relationship between throughput and channel allocation phase length.

transmission time being reduced and the saturation throughput decreasing. When the primary users' activity rate gets higher, fewer idle channels become available. Idle channels are therefore assigned soon when the channel allocation time is small. For example, the throughput reaches the maximum when the channel allocation phase length is short.

4.3. The Relationship between Throughput and Number of the Licensed Channels. The relationship between the number of licensed channels and throughput in the DAHCWNSMAC protocol is shown in Figure 7. The number of sending stations is 20 and the channel allocation phase length is $15 \mathrm{~ms}$. When the number of licensed channels increases, the saturation throughput increases under different primary users' activity rate, which means the proposed protocol can fully utilize licensed channels to communicate without interrupting primary users.

4.4. The Relationship between Throughput and Number of Sending Stations. Figure 8 shows the impact of the number of sending stations on the DAHCWNS-MAC protocol's throughput. The number of licensed channels is 20 and the channel allocation phase length is $15 \mathrm{~ms}$. As the number of sending stations increases, the throughput will increase and then remain stable when the primary users' activity rate is 0 . This is because the number of node pairs that can complete a handshake is larger than 20 when the number of licensed channels is 20. The saturation throughput will reach the maximum when all channels are assigned. After this, because there are no more available channels, an increase in the number of sending stations will not result in an increase in the throughput. So the throughput remains stable.

4.5. Comparison to the C-MMAC Protocol. Reference [19] proposes the C-MMAC (cognitive-multichannel MAC) protocol. This protocol is also a synchronous MAC protocol for

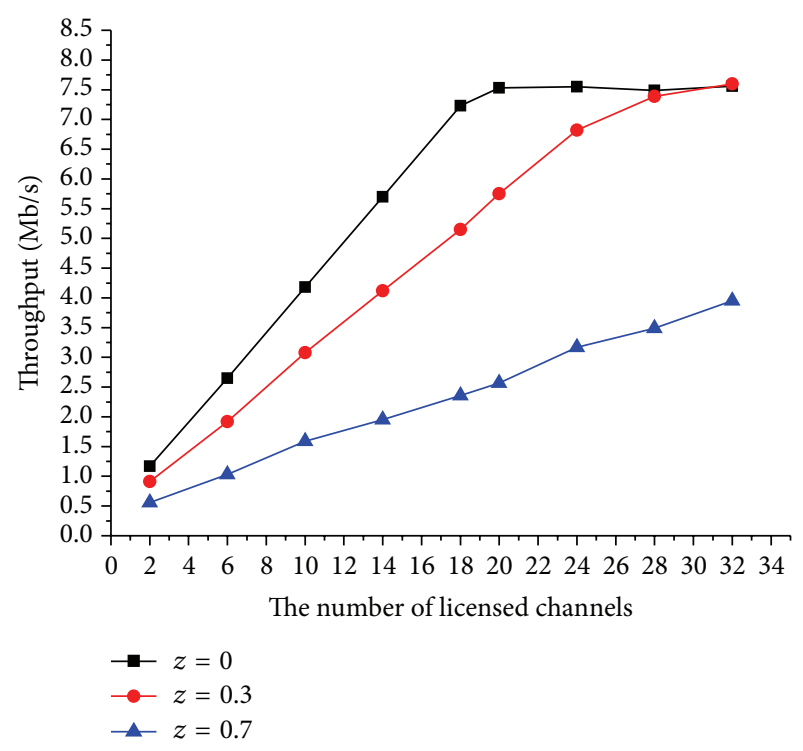

FIGURE 7: The relationship between throughput and number of licensed channels.

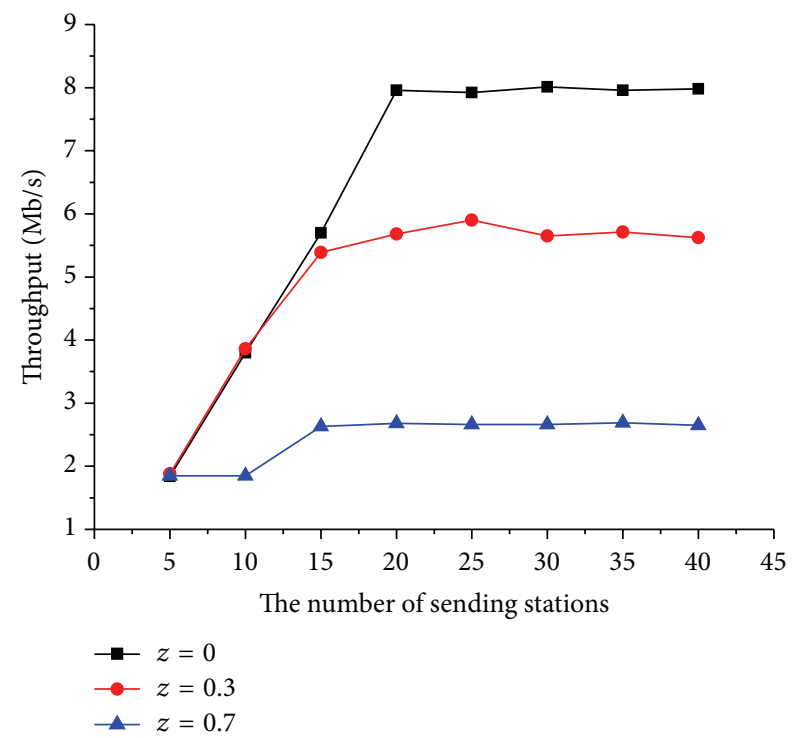

FIGURE 8: The relationship between throughput and number of sending stations.

cognitive radio network. The C-MMAC has some similarities with the DAHCWNS-MAC protocol: (1) The time frame of the C-MMAC protocol is divided into several parts (also include sensing phase, channel allocation phase, and data transmission phase) too and (2) all the nodes are strictly synchronous. The idea of designing DAHCWNS-MAC protocol is inspired by the C-MMAC protocol. However, the biggest difference between the two protocols is the network architecture. The C-MMAC protocol sets a single-hop network. The distance between two arbitrary nodes is within single-hop transmission range, and nodes can communicate directly. The network is self-organized, without infrastructure. Nodes have to sense the spectrum, exchange results, and negotiate 
channels by themselves. However, the protocol proposed in this paper is a hybrid network design. So this paper put these two protocols together to compare.

The two protocols are compared next through simulation. Since C-MMAC protocol is designed for the single-hop scenario, the scheme used in C-MMAC protocol is assumed to be a one-cell DAHCWNS-MAC protocol network. We compare the performance under the single-hop setting first (in one cell). The performance under a multiple cells network will be compared later.

4.5.1. Comparison in Single Cell. Nodes are placed randomly in one cell and communicate directly.

(1) 10 pairs of nodes are randomly placed in a cell, forming 10 communication streams. The number of licensed channels is 4. As shown in Figure 9 the throughput varies as the channel allocation phase length changes. When the primary user activity rate is 0 the C-MMAC protocol throughput is larger than that for the DAHCWNS-MAC at the beginning. This is because the C-MMAC protocol requires a three-way handshake (ATIM frame, ATIM-ACK frame, and ATIM-RES frame) to complete channel allocation while the DAHCWNSMAC protocol requires a four-way handshake (SEARCH frame, ANSWER frame, SETUP frame, and ACCEPT frame). Under the same channel allocation window the C-MMAC protocol allows more nodes to complete a handshake to get available channels, which results in higher throughput. When the channel allocation window gets larger it allows most nodes to complete a handshake. The C-MMAC protocol also adopts a competition scheme during the data transmission phase, which results in a longer time to send data packets than the DAHCWNS-MAC protocol. The C-MMAC throughput is therefore smaller than that of the DAHCWNS-MAC protocol.

(2) 10 pairs of nodes are randomly placed in a cell, forming 10 communication streams. The channel allocation window length is fixed at $30 \mathrm{~ms}$ which is sufficient for 10 pairs of nodes to complete a handshake to get available channels. As shown in Figure 10 the throughput varies as the number of licensed channels changes. When the primary user activity rate is 0 all licensed channels are idle and the control channel can also be used to transmit data. The throughput of the DAHCWNS-MAC protocol reaches the maximum when the number of licensed channels is 9 and then remains stable. However, the nodes must sense the spectrum by themselves in the C-MMAC protocol. Not all channels are detected in order to save power. Each node randomly selects a channel to sense and exchange its results with other nodes to obtain the entire sensing results. Whether all channels can be sensed depends on the number of nodes. According to [19], the probability that all channels can be detected is 0.95 when there are 10 licensed channels and 50 nodes. When the number of licensed channels is equal to the number of nodes the probability falls to 0.036 . This is why the C-MMAC throughput does not reach the maximum when the number of licensed channels increases to 9 (not all 9 channels have been detected). The maximal throughput does not come up until the number increases to 16 when the nodes can detect

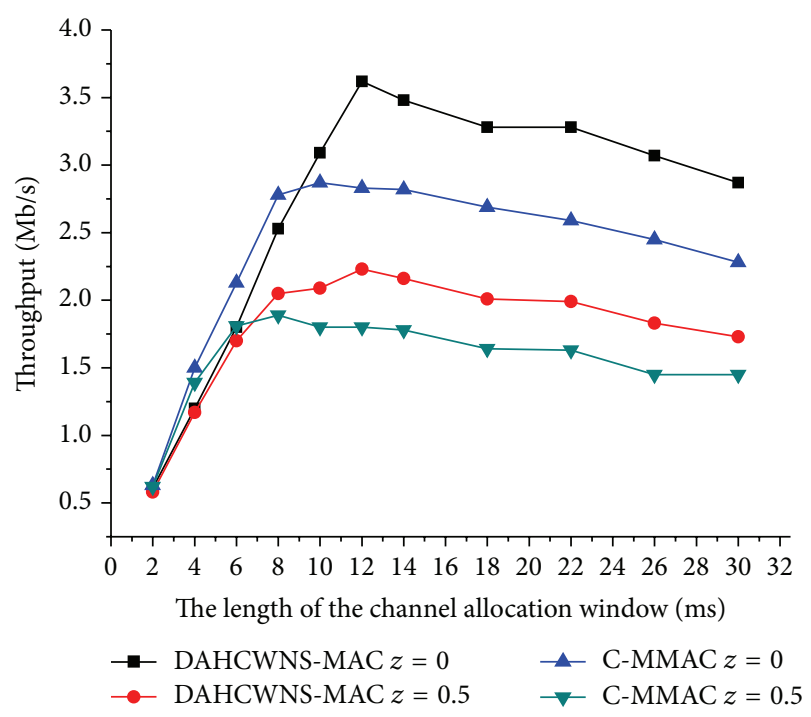

FIgURE 9: The comparison between the DAHCWNS-MAC protocol and the C-MMAC protocol as the channel allocation window length varies.

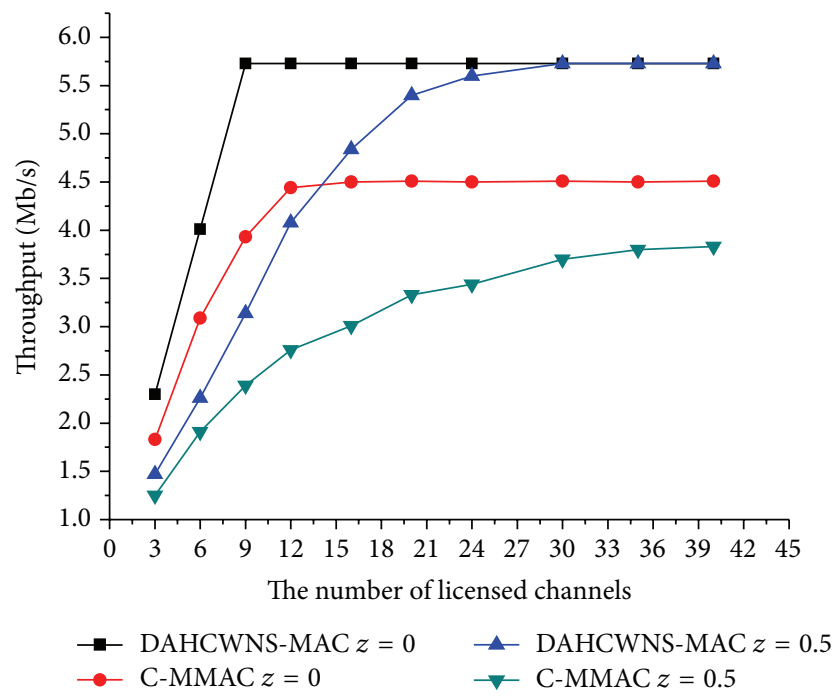

FIGURE 10: The comparison between the DAHCWNS-MAC protocol and the C-MMAC protocol as the number of licensed channels varies.

10 idle channels. The DAHCWNS-MAC protocol is free from this problem because distributed antennas undertake the spectrum sensing job and are able to detect all channels. The curve has the same trend when the primary user activity rate is 0.5 .

4.5.2. Comparison in Multiple Cells. The DAHCWNS-MAC protocol scheme is changed to seven cells while the C-MMAC protocol scheme remains the same.

(3) The number of nodes in the DAHCWNS-MAC protocol multiple cells scheme is more than seven times that of the C-MMAC single cell protocol. Assume that 35 pairs of nodes exist in the former scene and 5 pairs in the latter 


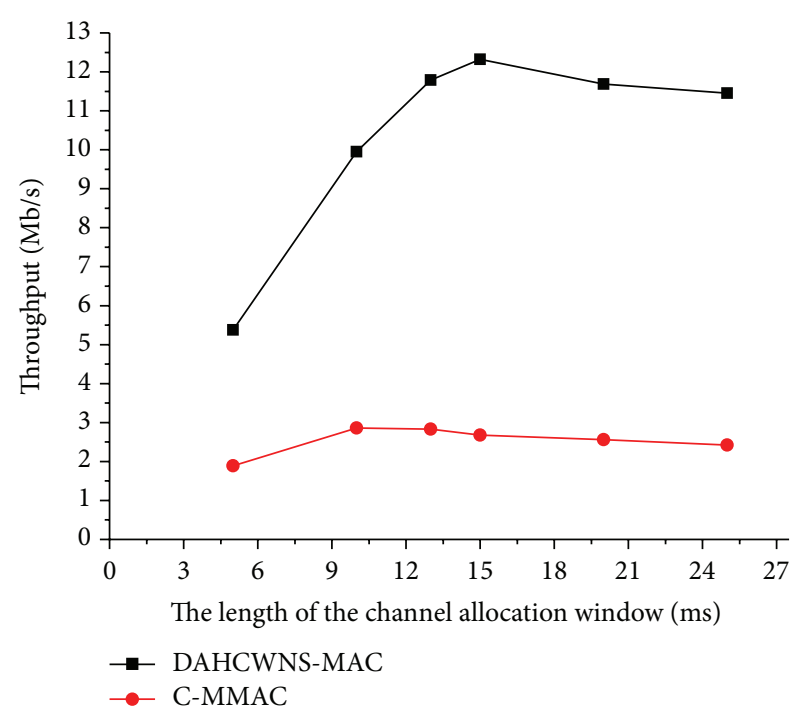

FIGURE 11: Comparison in multiple cells with different numbers of nodes.

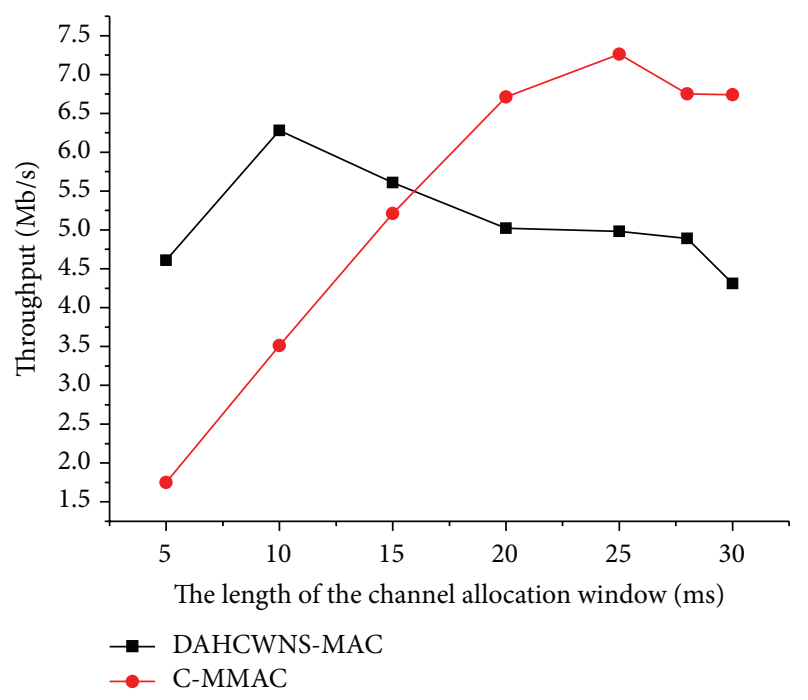

FIGURE 12: Comparison in multiple cells with the same number of nodes.

scheme. Ensure that nodes can be assigned channels as long as they complete a handshake. As shown in Figure 11, the DAHCWNS-MAC protocol throughput is not exactly seven times as large as that of the C-MMAC protocol. This is because the case nodes require distributed antennas to forward data which takes double the time of direct transmission.

(4) Assume 15 pairs of nodes are randomly placed in the two protocols, which means the number of nodes is the same in both schemes. Ensure that nodes can be assigned channels as long as they complete a handshake. As shown in Figure 12, the DAHCWNS-MAC protocol throughput is larger than that of the C-MMAC protocol when the channel allocation window length is relatively small. This is because operations in seven cells can be conducted in parallel, which leads to more nodes being able to complete a handshake. Since seven

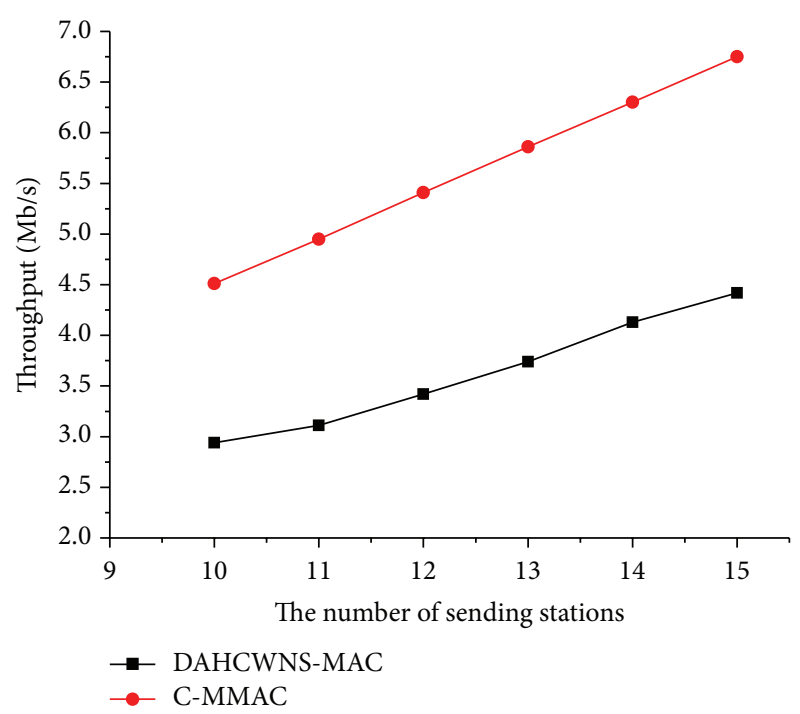

FIGURE 13: Comparison of multiple cells with the same number of nodes as the number of sending stations varies.

cells can work independently, 15 pairs of nodes can complete a handshake within $10 \mathrm{~ms}$ and the throughput reaches peak at this moment. After this moment the increase in channel allocation window length only leads to a decrease in the DAHCWNS-MAC protocol throughput. As the window becomes larger the number of nodes that finish a handshake becomes larger in C-MMAC. Since nodes can communicate directly, not like the DAHCWNS-MAC where antennas are required to forward data, the throughput becomes gradually higher than that of the DAHCWNS-MAC and reaches the maximum at $25 \mathrm{~ms}$.

(5) Assume the number of nodes in the two schemes is the same. The channel allocation window is fixed at $30 \mathrm{~ms}$. The number of licensed channels is sufficient. As shown in Figure 13, because the case exists where nodes need antennas to forward data in DAHCWNS-MAC, the throughput is absolutely less than that of the C-MMAC protocol where nodes are put together in a single cell.

\section{Conclusion}

The paper introduced distributed antennas into the cognitive wireless network. The cognitive wireless network is designed to be a heterogeneous network consisting of an ad hoc network with a sparse network with infrastructure. A distributed antenna based synchronous MAC protocol (DAHCWNSMAC protocol) is also presented for the proposed network. This protocol utilizes distributed antennas to sense the spectrum and transmit data, which can improve the sensing performance and increase network throughput. Every part of the protocol was described in detail and a mathematical model and performance simulation were presented. The proposed protocol combines the advantages of the ad hoc network and the network with infrastructure to fully utilize idle licensed channels to increase throughput. We compared the proposed protocol with the C-MMAC protocol to demonstrate that the 
DAHCWNS-MAC protocol can broaden the communication range and increase the network throughput compared with the original single-hop network at the cost of increasing antenna hardware costs.

The introduction of distributed antennas into spectrum sensing can fully utilize the spatial resources at the expense of increasing the antenna hardware costs. It can also overcome hidden/exposed terminal problems to a certain extent and improve sensing performance.

In addition to the spectrum sensing, we can further let the distributed antennas be used to locate the primary user and adopt different access methods according to positioning information. For example, the hybrid underlay/overlay access scheme can be adopted through power control, which can greatly improve the channel utilization ratio and increase network throughput.

Furthermore, in the DAHCCWNS-MAC protocol the center allocates the same channel to communication pair which is not flexible enough. In future work we can take the channel state information into consideration and allocate different channels to sending node and receiving node, respectively, according to in-time channel state to further improve network performance.

This paper focused on how the distributed antenna can be used in data transmission and did not explore its usage in spectrum sensing and positioning. In future studies we can continue to study on these aspects to complete the DAHCWNS-MAC protocol.

\section{Conflict of Interests}

The authors declare that there is no conflict of interests regarding the publication of this paper.

\section{Acknowledgments}

The work presented in this paper was partially supported by 2011 National Natural Science Foundation of China (Grant no. 61172097), 2014 National Natural Science Foundation of China (Grant no. 61371081), and 2012 Natural Science Foundation of Fujian (Grant no. 2012J01424).

\section{References}

[1] P. Yue, X. Yi, and Z.-J. Liu, "A novel wireless network architecture and its radio frequency assignment mechanism for WLAN based on distributed antenna system using radio over free space optics," in Proceedings of the International Conference on Information Science and Technology (ICIST '11), pp. 488-492, March 2011.

[2] Z. Xu, C. Zhou, and J. Wang, "A novel cell architecture based on distributed antennas for mobile WiMAX systems," in Proceedings of the 4th IEEE International Conference on Circuits and Systems for Communications (ICCSC '08), pp. 172-176, May 2008.

[3] D. J. Thomson, "Spectrum estimation and harmonic analysis," Proceedings of the IEEE, vol. 70, no. 9, pp. 1055-1096, 1982.

[4] S. Haykin, D. J. Thomson, and J. H. Reed, "Spectrum sensing for cognitive radio," Proceedings of the IEEE, vol. 97, no. 5, pp. 849-877, 2009.
[5] Y. Zeng, C. L. Koh, and Y.-C. Liang, "Maximum eigenvalue detection: theory and application," in Proceedings of the IEEE International Conference on Communications (ICC '08), pp. 4160-4164, Beijing, China, May 2008.

[6] Y. Zeng and Y.-C. Liang, "Eigenvalue-based spectrum sensing algorithms for cognitive radio," IEEE Transactions on Communications, vol. 57, no. 6, pp. 1784-1793, 2009.

[7] A. Taherpour, M. Nasiri-Kenari, and S. Gazor, "Multiple antenna spectrum sensing in cognitive radios," IEEE Transactions on Wireless Communications, vol. 9, no. 2, pp. 814-823, 2010.

[8] R. Zhang, T. J. Lim, Y. C. Liang, and Y. Zeng, "Multi-antenna based spectrum sensing for cognitive radios: a GLRT approach," IEEE Transactions on Communications, vol. 58, no. 1, pp. 84-88, 2010.

[9] H. Yao, Research on the Spectrum Sensing and Resource Allocation in Cognitive Radio Networks, Beijing University of Posts and Telecommunications, 2011.

[10] C. Zhao, L. Huang, Z.-L. Gao, S. Zhou, D. Guo, and H.-C. Chao, "performance analysis of the multiple antenna asynchronous cognitive MAC protocol in cognitive radio network for IT convergence," Intelligent Automation and Soft Computing, vol. 20, no. 1, pp. 61-75, 2014.

[11] E. Y. Kang, H. Park, and J. Chae, "A hybrid message delivery scheme for improving service discovery in mobile ad-hoc networks," Journal of Internet Technology, vol. 13, no. 6, pp. 879890, 2012.

[12] Y.-X. Lai, C.-F. Lai, Y.-M. Huang, and H.-C. Chao, "Multiappliance recognition system with hybrid SVM/GMM classifier in ubiquitous smart home," Information Sciences, vol. 230, pp. 39-55, 2013.

[13] L. Zhou, H.-C. Chao, and A. V. Vasilakos, "Joint forensicsscheduling strategy for delay-sensitive multimedia applications over heterogeneous networks," IEEE Journal on Selected Areas in Communications, vol. 29, no. 7, pp. 1358-1367, 2011.

[14] C.-W. Chiang, "Two novel genetic operators for task matching and scheduling in heterogeneous computing environments," Journal of Internet Technology, vol. 13, no. 5, pp. 773-784, 2012.

[15] B. Liu, Z. Liu, and D. Towsley, "On the capacity of hybrid wireless networks," in Proceedings of the 22nd Annual Joint Conference on the IEEE Computer and Communications Societies, vol. 2, pp. 1543-1552, April 2003.

[16] R. S. Chang, W. Y. Chen, and Y. F. Wen, "Hybrid wireless network protocols," IEEE Transactions on Vehicular Technology, vol. 52, no. 4, pp. 1099-1109, 2003.

[17] G. Bianchi, "Performance analysis of the IEEE 802.11 distributed coordination function," IEEE Journal on Selected Areas in Communications, vol. 18, no. 3, pp. 535-547, 2000.

[18] N. Liu, Wireless Local Area Networks (WLAN)-Principle,Technique and Application, Xidian University Press, Xi'an, China, 2007.

[19] M. Yu, The Study of Cognitive Multi-Channel MAC Protocol Based on Spectrum Sensing, 2009. 

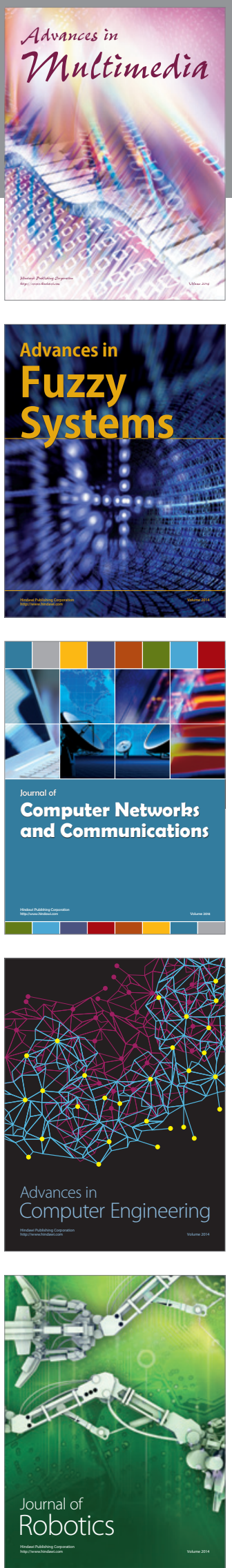

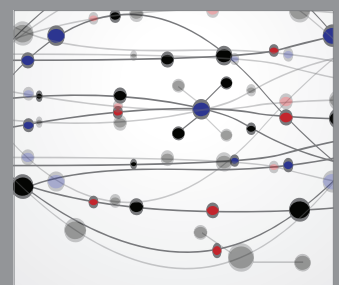

The Scientific World Journal
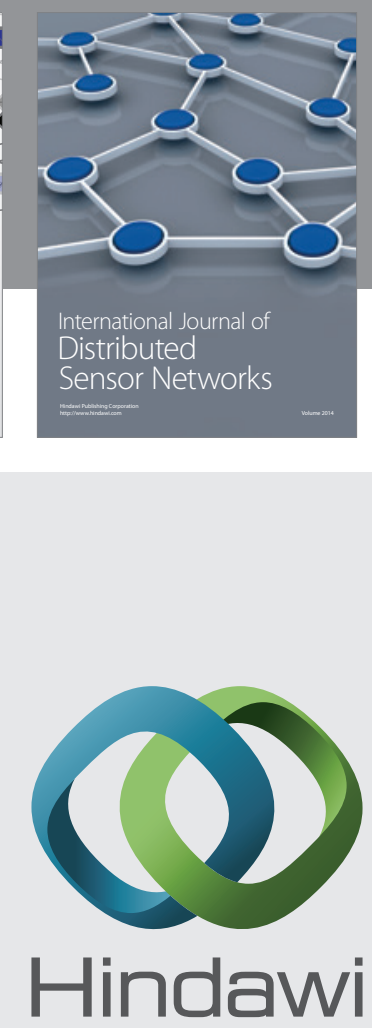

Submit your manuscripts at

http://www.hindawi.com
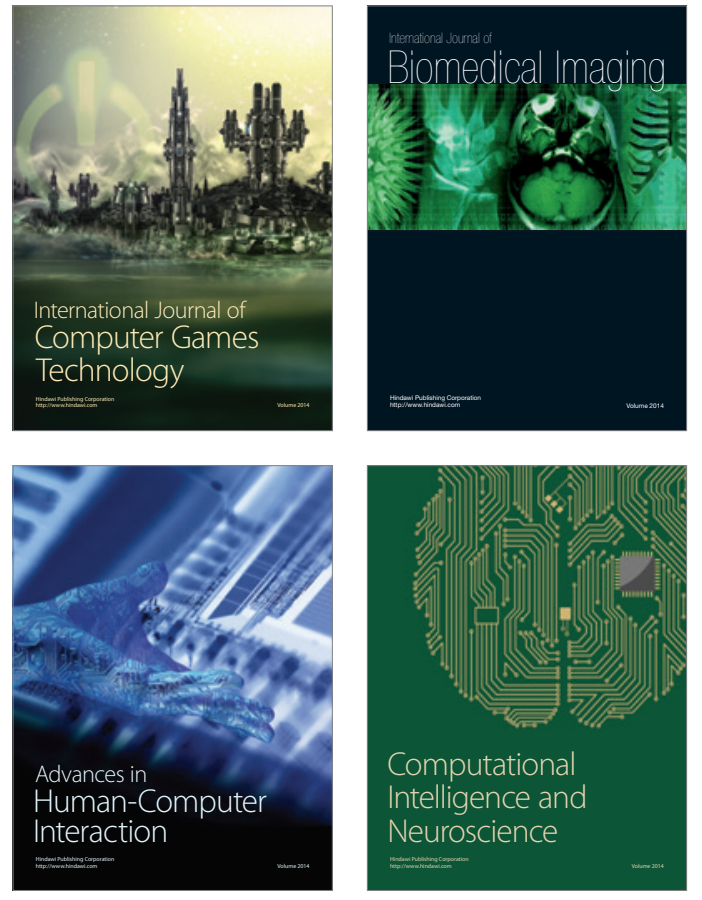
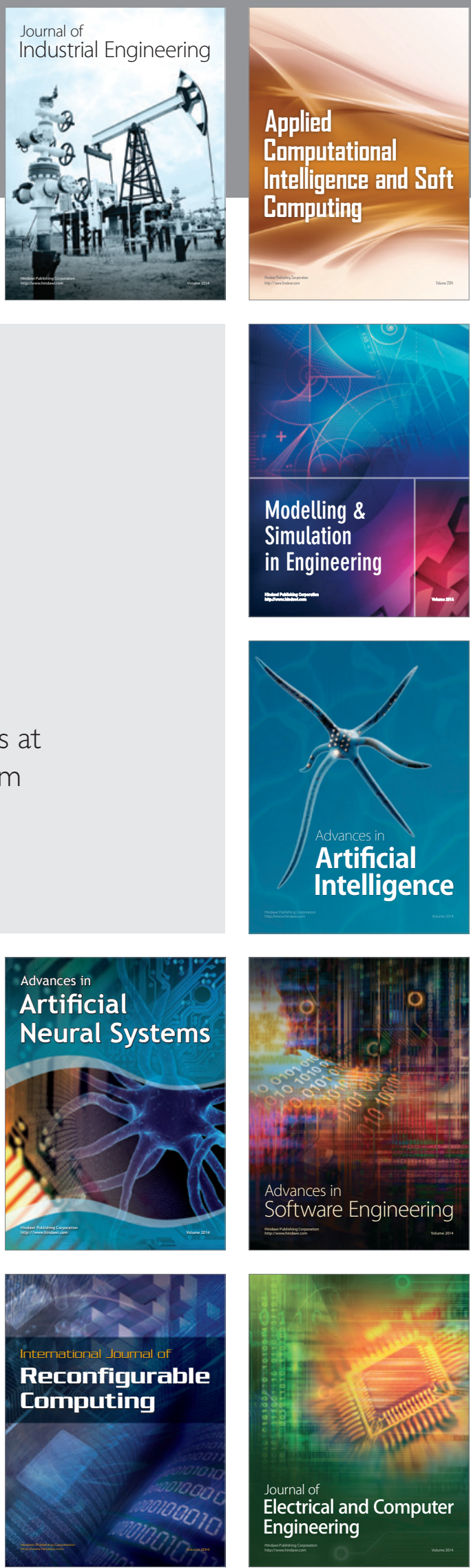